\title{
Hemorroidectomia Convencional Versus Hemorroidopexia Mecânica (PPH). Estudo Retrospectivo de 253 Casos
}

\section{Conventional Hemorrhoidectomy Versus Stapled Hemorrhoidopexy (PPH). A Retrospective Study of 253 Patients}

\author{
RAPHAEL MARIANELLI ${ }^{1}$, STÊNNIO PABLO GONÇALVES MACHADO $^{1}$, MARISTELA GOMES DE ALMEIDA $^{2}$,

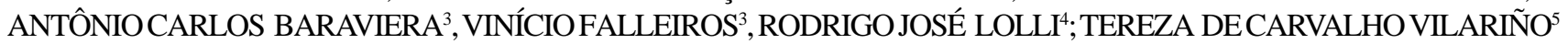 \\ ${ }^{1}$ Residente de Coloproctologia do Hospital do Servidor Público Municipal de São Paulo (HSPM-SP), ${ }^{2}$ Mestra e \\ Doutora em Coloproctologia, médica assitente junto ao Departamento de Coloproctologia do HSPM-SP, ${ }^{3}$ Médico \\ assistente junto ao Departamento de Coloproctologia do HSPM-SP, ${ }^{4}$ Acadêmico de medicina da UNIMES, ${ }^{5}$ Mestra em \\ Coloproctologia e Coordenadora do Departamento de Coloproctologia do HSPM-SP.
}

\begin{abstract}
MARIANELLI R; MACHADO SPG; ALMEIDA MG; BARAVIERAAC; FALLEIROS V; LOLLI RJ; VILARIÑO TC. Hemorroidectomia Convencional Versus Hemorroidopexia Mecânica (PPH). Estudo Retrospectivo de 253 Casos. Rev bras Coloproct, 2009;29(1): 030-037.

RESUMO: O objetivo deste estudo foi rever a experiência do serviço de coloproctologia do HSPM-SP no tratamento cirúrgico da doença hemorroidária. Estudou-se retrospectivamente 253 pacientes portadores de hemorroidas submetidos à cirurgia no período de 10/05/2004 a 31/12/2007. Foram estabelecidos dois grupos: Grupo 1) Pacientes operados pela técnica convencional (212); Grupo 2) Pacientes operados pelo PPH (41). Estudou-se, pela revisão de prontuários, as características epidemiológicas, indicações, resultados e complicações de cada grupo. Entre os 253 pacientes analisados, 170 eram mulheres, com média de idade de 50 anos. O tempo operatório médio foi de 52 minutos para o grupo 1, e 31 minutos para o grupo 2 (p=0,0001). 0 tempo médio de alta ambulatorial foi de 10 e 6 semanas para o grupo 1 e 2 , respectivamente (p=0,021). As complicações totalizaram 28 casos no grupo 1 , e 3 casos no grupo 2 (p>0,05). Cerca de 13 casos no grupo 1, e 1 caso no grupo 2 necessitaram de reintervenção cirúrgica. Neste serviço a técnica mais utilizada foi a hemorroidectomia convencional. O método do PPH apresentou tempo operatório mais curto, e recuperação pós-operatória mais breve. Houve uma maior tendência a complicações tardias e recidivas com o grupo de hemorroidectomia convencional, porém sem significância estatística.
\end{abstract}

Descritores: Hemorroidas, hemorroidectomia, hemorroidopexia, PPH, complicações.

\section{INTRODUÇÃO}

O tratamento das hemorróidas pode ser classificado em três categorias: 1) modificação da dieta e estilo de vida; 2) procedimentos ambulatoriais não cirúrgicos; 3) tratamento cirúrgico ${ }^{1,2}$.

As modificações de dieta e estilo de vida são aplicáveis a todos os pacientes, sendo muitas vezes o único tratamento necessário nos pacientes com doença de $1^{\circ}$ e $2^{\circ}$ grau. Consistem numa adequada ingestão de líquidos e fibras, o que pode ser obtido com a simples modificação de hábitos alimentares ou com ajuda de su- plementos de fibra dietética (psyllium, plantago ovata, ágar-ágar, metilcelulose, cálcio policarbofil) disponíveis sob diferentes nomes comerciais. Modificações dos hábitos evacuatórios e de higiene pessoal, evitando uso do papel higiênico, também são recomendáveis ${ }^{1,2}$.

Uma série de procedimentos ambulatoriais nãooperatórios está descrita na literatura, podendo-se citar entre eles: ligadura elástica, escleroterapia, crioterapia, fotocoagulação por infravermelho, diatermia e eletrocoagulação. Estas técnicas podem ser utilizadas em doença de $1^{\circ}$ grau, alguns casos de $2^{\circ}$ grau e casos bem selecionados de $3^{\circ} \mathrm{grau}^{1-4}$. 
Hemorroidectomia Convencional Versus Hemorroidopexia Mecânica (PPH). Estudo Retrospectivo de 253 Casos

Raphael Marianelli e Cols.
A indicação clássica de cirurgia no tratamento das hemorroidas acontece nos casos de $3^{\circ}$ e $4^{\circ}$ grau, estimando-se em $5 \%$ a $10 \%$ a necessidade do tratamento operatório. A recorrência é incomum após tratamento cirúrgico, e este sem dúvida é o método terapêutico mais eficaz ${ }^{1,3-5}$.

O princípio fundamental da hemorroidectomia é a ressecção dos coxins ou mamilos hemorroidários, com ou sem a ligadura do pedículo vascular. Apesar dos inúmeros epônimos utilizados para sua descrição, existem basicamente quatro técnicas: 1) hemorroidectomia aberta, descrita por Milligan e Morgan $^{6}$; 2) hemorroidectomia fechada, descrita por Ferguson $^{7}$; 3) hemorroidectomia circular, descrita por Whitehead $^{8}$; 4) hemorroidectomia mista (combinação de técnicas). Alguns cirurgiões propuseram aperfeiçoamentos para as técnicas originais, como Obano", Parks $^{10}$, Sokol ${ }^{11}$ e Ruiz Moreno ${ }^{12}$, sem caracterizar uma nova técnica.

Apesar de diferentes proposições técnicas a hemorroidectomia têm índice de complicações considerável, variando entre 3,1\% e $12 \% \%^{2,13-24}$. Dentre as complicações mais comuns estão: dor local, retenção urinária, hemorragia, estenose anal, ferida fissuróide, fístula perianal, incontinência anal e recorrênciaa ${ }^{1-3,5,13-25}$.

Recentemente um novo método cirúrgico para o tratamento das hemorroidas foi apresentado por Lon$\mathrm{go}^{26}$. Inicialmente denominado de forma errônea como hemorroidectomia mecânica, este método difere drasticamente da hemorroidectomia convencional descrita acima. Consiste basicamente na ressecção de faixa circular de mucosa do reto distal, acima dos mamilos hemorroidários, com o auxílio de um grampeador endoluminal circular. Nesta técnica não se realiza uma hemorroidectomia e sim uma fixação dos coxins hemorroidários em sua posição anatômica. Em 2003, um consenso internacional no assunto propôs a modificação da nomenclatura para Hemorroidopexia Mecânica, além de padronizar a técnica e definir contraindicações ${ }^{27}$.

A hemorroidopexia mecânica, por não produzir incisões e feridas sobre o anoderma do canal anal, produz recuperação pós-operatória mais rápida e com menos dor, propiciando o retorno precoce as atividades cotidianas. No entanto, complicações sérias têm sido relatadas como perfuração retal, sepsis retroperitoneal e abscesso pélvico. Outras complicações mais comuns e menos graves incluem trombose hemorroidária, sangramento da linha de grampeamento, recidiva, estenose, incontinência e fissura anal. Os índices gerais de complicação variam muito na literatura, com valores entre $3 \%$ e $31 \%{ }^{1,3,4,28-43}$.

\section{OBJETIVO}

O objetivo deste estudo é rever a experiência do Serviço de Coloproctologia do Hospital do Servidor Público Municipal de São Paulo (HSPM) no tratamento cirúrgico da doença hemorroidária, observando e comparando as características epidemiológicas, indicações, complicações e resultados das técnicas de hemorroidectomia convencional e hemorroidopexia mecânica (PPH).

\section{CASUÍSTICA E MÉTODOS}

Foram revisados 283 prontuários de pacientes portadores de doença hemorroidária submetidos ao tratamento cirúrgico entre 10/05/2004 e 31/12/2007. Pacientes portadores de hemorroidas externas ou mistas, trombose hemorroidária, hemorroidectomia prévia, fissura, fístula ou condiloma anal associados foram excluídos da pesquisa, chegando a um total de 253 pacientes portadores de hemorroidas internas isoladas. Esta população foi dividida em dois grupos: Grupo 1) Pacientes operados pela técnica de Hemorroidectomia Convencional $(n=212)$; Grupo 2) Pacientes operados pela técnica de Hemorroidopexia Mecânica (n=41). No Grupo 1 as técnicas utilizadas foram a de Milligan-Morgan ${ }^{6}$, Ferguson $^{7}, \mathrm{Obano}^{9}$ ou Técnica Mista (Tabela 1). No Grupo 2 os pacientes foram operados conforme a técnica descrita por Longo e preconizada pelo Consenso Internacional ${ }^{26,27}$. Foram estudadas de forma retrospectiva, através da revisão de prontuários, as características epidemiológicas, indicações, complicações, necessidade de reintervenção cirúrgica, tempo operatório, alta ambulatorial e seguimento para cada grupo. Os dados

Tabela 1 - Distribuição das Hemorroidectomias por técnica utilizada.

\begin{tabular}{lrc}
\hline Hemorroidectomia & $\mathbf{N}$ & $\mathbf{( \% )}$ \\
\hline Milligan-Morgan & 187 & $(88,2)$ \\
Ferguson & 1 & $(0,5)$ \\
Obano & 6 & $(2,8)$ \\
Mista & 18 & $(8,5)$ \\
Total & $\mathbf{2 1 2}$ & - \\
\hline
\end{tabular}


foram coletados por um único pesquisador, e analisados somente após a documentação de todos os prontuários. Os resultados e complicações de ambos os grupos foram comparados e verificados quanto à significância estatística através do teste exato de Fischer, com auxílio de software estatístico (MedCalc® versão 9.6.4.0).

\section{RESULTADOS}

Quanto à técnica utilizada, 212 pacientes (83.8\%) foram submetidos a Hemorroidectomia Convencional (Grupo 1), enquanto 41 pacientes $(16.2 \%)$ a Hemorroidopexia Mecânica ou PPH (Grupo 2).

Entre os 253 pacientes analisados 170 eram mulheres $(67.2 \%)$ e 83 homens (32.8\%). A média de idade foi de 50 anos (19 a 78 anos), com 63\% dos indivíduos na faixa entre 41 e 60 anos. A tabela 2 compara as diferenças entre os 2 grupos.

A indicação dos procedimentos foi avaliada pela classificação da doença hemorroidária. Em 36 casos (14.2\%) as hemorróidas foram classificadas como de $2^{\circ}$ Grau com sangramento, em 180 casos $(71.2 \%)$ como de $3^{\circ}$ Grau e em 37 casos (14.6\%) como de $4^{\circ}$ Grau. As diferenças entre Grupo 1 e Grupo 2 estão resumidas na tabela 3 .

O tempo operatório médio por procedimento (Gráfico 1) foi de 52 minutos (25 a 120 minutos) no
Grupo 1 e de 31 minutos (20 a 65 minutos) no Grupo 2 $(\mathrm{p}=0,0001)$.

A data de alta ambulatorial foi definida como a última consulta relacionada ao procedimento cirúrgico no período pós-operatório. O tempo médio de alta ambulatorial (Gráfico 2) calculado para o Grupo 1 foi de 10 semanas, enquanto no Grupo 2 foi de 6 semanas $(\mathrm{p}=0,021)$.

Para melhor entendimento, as complicações foram divididas em precoces e tardias. Como complicação precoce destacamos a fissura anal aguda e a retenção urinária. No Grupo 1 encontramos 9 casos (4.2\%) de retenção urinária, enquanto no Grupo 2 houve 1 caso $(2.4 \%)$ de retenção urinária e 1 caso $(2.4 \%)$

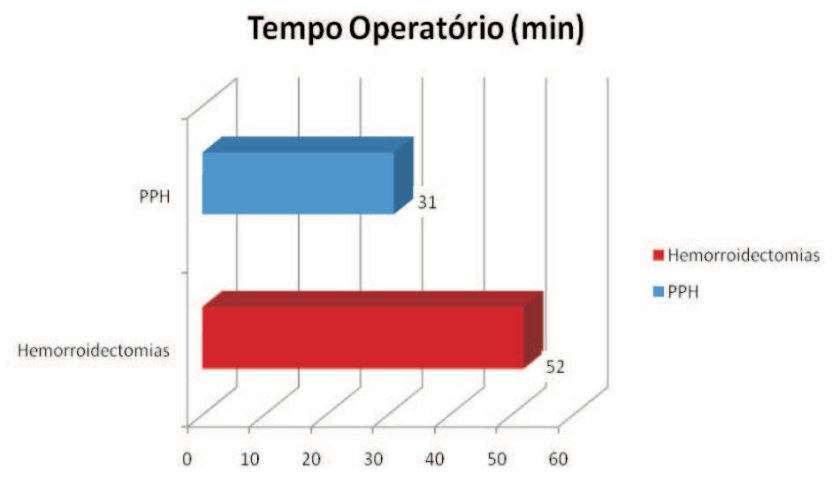

Gráfico 1 - Tempo Operatório Médio $(p=0,0001)$.

Tabela 2 - Distribuição por faixa etária.

\begin{tabular}{lccrrrr}
\hline Faixa Etária & \multicolumn{2}{c}{ Hemorroidectomias } & & PPH & \multicolumn{2}{c}{ Total } \\
\cline { 2 - 7 } & $(\mathbf{N})$ & $\mathbf{\%}$ & $(\mathbf{N})$ & $\mathbf{\%}$ & $\mathbf{( N )}$ & \% \\
\hline $11-20$ & 2 & 0,9 & 0 & 0 & 2 & 0,8 \\
$21-30$ & 6 & 2,8 & 1 & 2,5 & 7 & 2,8 \\
$31-40$ & 34 & 16,0 & 6 & 14,6 & 40 & 15,8 \\
$41-50$ & 73 & 34,5 & 10 & 24,4 & 83 & 32,8 \\
$51-60$ & 62 & 29,3 & 14 & 34,1 & 76 & 30,0 \\
$61-70$ & 28 & 13,2 & 7 & 17,1 & 35 & 13,8 \\
\hline
\end{tabular}

Tabela 3 - Indicações Cirúrgicas.

\begin{tabular}{|c|c|c|c|c|c|c|}
\hline \multirow[t]{2}{*}{$\overline{\text { Grau }}$} & \multicolumn{2}{|c|}{ Hemorroidectomias } & \multicolumn{2}{|c|}{$\mathbf{P P H}$} & \multicolumn{2}{|c|}{ Total } \\
\hline & $(\mathrm{N})$ & $\%$ & $(\mathbf{N})$ & $\%$ & $(\mathbf{N})$ & $\%$ \\
\hline II & 34 & 16,0 & 2 & 4,9 & 36 & 14,2 \\
\hline III & 154 & 72,6 & 26 & 63,4 & 180 & 71,2 \\
\hline IV & 24 & 11,4 & 13 & 31,7 & 37 & 14,6 \\
\hline
\end{tabular}


de fissura anal aguda. Todos os casos de retenção urinária foram tratados com sondagem vesical de alívio tendo alta hospitalar no $1^{\circ}$ dia do pós-operatório sem maiores intercorrências. $\mathrm{O}$ caso de fissura aguda pós-PPH foi tratado conservadoramente com banho de assento, orientação higienodietética, diltiazen tópico a $2 \%$ e analgésicos durante 2 meses, e como este permanecia sintomático foi indicada esfincterotomia lateral interna com resolução do quadro. Nesta série não houve casos de sangramento anal volumoso (definido como necessidade de reintervenção) ou trombose hemorroidária.

As complicações tardias encontradas foram: fístula anal, incontinência esfincteriana, recidiva do prolapso, estenose anal e ferida fissuroide, definida aqui como a não cicatrização da ferida operatória 2 meses após o procedimento. No Grupo 1 a ocorrência de complicações tardias foi de 19 casos (9\%), com 7 (3.3\%) feridas fissuroides, 5 (2.3\%) fístulas anais, 4 casos (1.9\%) de estenose anal, 2 (1.0\%) recidivas de prolapso e $1(0.5 \%)$ incontinência anal. No Grupo 2 houve apenas 1 caso $(2.4 \%)$ de incontinência anal como complicação tardia.

Dos sete casos de ferida fissuroide, três necessitaram correção cirúrgica com fissurectomia, ampliação da ferida externa e cicatrização por segunda intenção. Em quatro pacientes foi adotada conduta conservadora, evoluindo com cicatrização completa ao fim de 24, 25, 30 e 32 semanas de pós-operatório.

Entre os cinco casos de fístula anal, três foram diagnosticados nos primeiros seis meses de pós-operatório (dois casos com 3 meses e um com 5 meses) e dois após um ano de cirurgia ( 1 ano e três meses e 1 ano e 6 meses, respectivamente). Todos os casos foram operados pela técnica de fistulotomia, sendo que um deles se tratava de fístula transesfincteriana superficial e o restante de trajeto subcutâneo superficial compatível com vício de cicatrização.

Os casos de recidiva foram diagnosticados entre 10 e 15 meses de pós-operatório. Como o primeiro queixava-se de sangramento persistente, foi indicada nova operação pela técnica de MilliganMorgan $^{6}$. O outro paciente era assintomático não necessitando reoperação.

Todos os quatro casos de estenose anal póscirúrgica foram classificados como de forma anelar e da margem anal, sendo que um deles apresentava fissura associada. O diagnóstico foi feito entre 3 e 13 meses de pós-operatório. Em todos pacientes foi indicada anoplastia pela técnica de Musiari ${ }^{44}$, com bons resultados e alívio dos sintomas. A correção aconteceu entre 3 e 25 meses de pós-operatório.

Nenhum dos dois pacientes com queixa de incontinência anal necessitou de correção cirúrgica, apresentando Cleveland Clinic Incontinence Score (CCIS) de 2 (paciente do grupo 1) e 4 (paciente do grupo 2). Quando questionados, nenhum deles se referiu a sintomas no pré-operatório, no entanto o CCIS não foi aplicado aos mesmos nesta fase. O diagnóstico aconteceu entre 2 e 3 meses de pós-operatório.

No geral a taxa de reoperação no Grupo 1 foi de $6.1 \%$, enquanto no Grupo 2 foi de $2.4 \%$. As diferenças entre os dois grupos quanto as complicações não obtiveram significância estatística. A tabela 4 e o gráfico 3 sintetizam as complicações e reoperações referidas.

\section{DISCUSSÃO}

Este artigo é um estudo retrospectivo baseado na análise de prontuários de pacientes portadores de

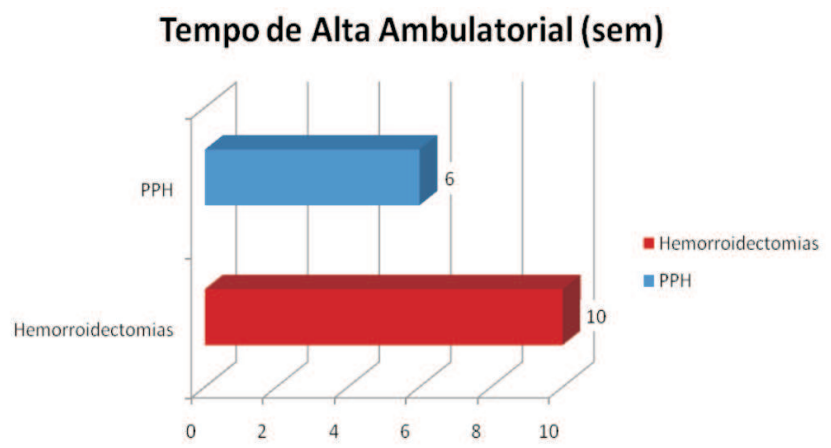

Gráfico 2 - Tempo Médio de Alta Ambulatorial $(p=0,021)$.

\section{Complicações Anais vs. Reoperações}

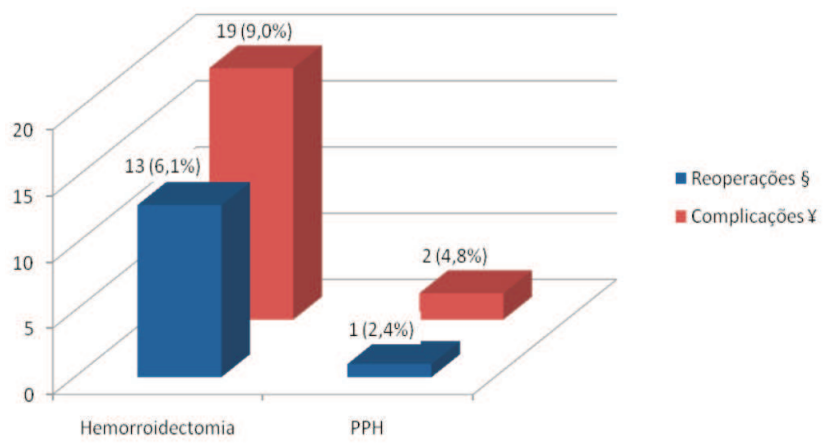

Gráfico 3 - Total de Complicações Anais e Reoperações por Grupos. $\S \mathrm{p}=0,54 ; ¥ \mathrm{p}=0,69$. 
Tabela 4 - Complicações Pós-Operatórias.

\begin{tabular}{|c|c|c|c|}
\hline$\overline{\text { Complicações }}$ & $\begin{array}{c}\text { Hemorroidectomias } \\
(\mathrm{n}=\mathbf{2 1 2})\end{array}$ & $\begin{array}{c}\text { PPH } \\
(n=41) \\
\end{array}$ & $p$ \\
\hline Precoces & $9 \quad(4,2 \%)$ & $1(2,4 \%)$ & 0,69 \\
\hline Retenção Urinária & $9 \quad(4,2 \%)$ & $1 \quad(2,4 \%)$ & 1,00 \\
\hline Fissura Aguda & - & $1 \quad(2,4 \%)$ & 1,16 \\
\hline Tardias & $19(9,0 \%)$ & $1 \quad(2,4 \%)$ & 0,21 \\
\hline Ferida Fissuroide & $7 \quad(3,3 \%)$ & 0 & 1,00 \\
\hline Fístula Perianal & $5 \quad(2,3 \%)$ & 0 & 0,60 \\
\hline Estenose Anal & $4 \quad(1,9 \%)$ & 0 & 1,00 \\
\hline Recidiva & $2 \quad(1,0 \%)$ & 0 & 1,00 \\
\hline Incontinência & $1 \quad(0,5 \%)$ & $1 \quad(2,4 \%)$ & 0,29 \\
\hline
\end{tabular}

doença hemorroidária, operados em decorrência de seus sintomas. As informações foram obtidas de acordo com o registro dos prontuários, não sendo possível re-examinar os pacientes.

Todo o esforço foi feito para tornar os grupos de estudo o mais homogêneo possível, estabelecendo critérios de exclusão já apresentados anteriormente. Apesar disso, permanecem diferenças importantes entre os dois grupos, sobretudo quanto ao número de pacientes. Além do que, o percentual de indivíduos com doença de grau avançado (III e IV) foi maior no grupo de hemorroidopexia, bem como, a média de idade mais avançada.

Mesmo assim, alguns aspectos tiveram significância estatística. A hemorroidopexia foi associada a tempo operatório médio mais curto (31 min vs. 52 min, $p<0,0001)$ e recuperação mais breve, avaliada indiretamente pelo tempo de alta ambulatorial ( $6 \mathrm{se}-$ manas vs. 10 semanas, $\mathrm{p}<0,021$ ).

No grupo de hemorroidectomia convencional (grupo 1), o índice geral de 9\% para complicações orificiais pós-operatórias, está de acordo com o descrito na literatura médica, variando entre $3,1 \%$ e $12 \%{ }^{2,13-24,28}$

Apesar dos relatos de hemorragia pós-operatória ser em torno de $0,03 \%$ a $6,0 \%{ }^{13-24,28}$, em nossa série não observamos necessidade de reintervenção, seja ela clínica ou cirúrgica, relacionadas a esta queixa. Além disso, foi observado um percentual relativamente elevado de fístulas anais pós-hemorroidectomia, totalizando 2,3\% dos casos, sendo que na maioria das séries este é um achado não usual, correspondendo no máximo a $1 \%$ do total ${ }^{1,4,28}$. O restante das complica- ções estão de acordo com a literatura em questão ${ }^{1,2,4,5,13-24,28}$.

No grupo de hemorroidopexia mecânica (grupo 2) encontramos índice geral de 4,8\% para complicações orificiais pós-operatórias. Na literatura encontramos relatos bem variáveis de complicações, oscilando entre $3 \%$ e $31 \% \%^{1,3,4,28-43}$. Recentemente, $\mathrm{Ravo}^{41}$, publicou uma revisão de 1.107 pacientes tratados por PPH em 20 centros italianos, revelando taxa geral de $15 \%$. Talvez esta disparidade de resultados se justifique por uma excessiva valorização de sinais e sintomas transitórios, como dor e sangramento, por parte de uns ${ }^{29,33,41}$, e sua não valorização por parte de ou$\operatorname{tros}^{31,32}$. O pequeno número de pacientes no grupo do PPH compromete uma análise comparativa de complicações específicas com outras séries. Por exemplo, as taxas de fissura anal e incontinência relatadas por $\mathrm{Ravo}^{41}$ são $0,8 \%$ e $0,2 \%$ respectivamente, enquanto em nossa análise encontramos 1 caso de cada $(2,4 \%)$.

A única complicação não anal avaliada foi a retenção urinária, que no grupo 1 foi de $4,2 \%$ e no grupo 2 de $2,4 \%(p=0,59)$. Revisões sistemáticas do assunto também mostram tendência de menor retenção com hemorroidopexia, entretanto, também sem significância estatística ${ }^{42,43}$.

Apesar da tendência a um maior número de complicações tardias com o grupo de hemorroidectomias, não observamos dados com significância estatística. Nas grandes metanálises do assunto $^{34,35,38,42,43}$ também não foram encontrados diferenças quanto a complicações anais pós operatórias, a não ser quanto à recidiva da doença, que foi sempre menor no grupo de hemorroidectomia convencional. No 
Rev bras Coloproct Janeiro/Março, 2009
Hemorroidectomia Convencional Versus Hemorroidopexia Mecânica (PPH). Estudo Retrospectivo de 253 Casos

Raphael Marianelli e Cols.
Vol. 29 entanto, o grupo de hemorroidopexia mecânica obteve melhores resultados quanto à recuperação e à dor pósoperatória, menor internação hospitalar e retorno mais rápido às atividades cotidianas.

Quanto à necessidade de reintervenção cirúrgica, é difícil encontrarmos na literatura dados específicos para comparação. Recentemente, $\mathrm{Cruz}^{2}$ publicou revisão de 2.417 hemorroidectomias com taxa de reoperação de aproximadamente $3,0 \%$. Em contrapartida, encontrou-se $6,1 \%$ de reintervenções cirúrgicas pós-hemorroidectomia neste estudo. Tal disparidade talvez se justifique pelo elevado número de fístulas anais encontradas, que neste estudo foi a principal causa de reoperação, fato não observado em outras séries, como já discutido anteriormente. Já no grupo de hemorroidopexia $2,4 \%$ dos pacientes ( 1 caso) foram reoperados. A literatura mostra dados bem conflitantes no que se refere a reintervenção cirúrgica pós-PPH, com índice variando entre $4,1 \%$ e $26,7 \%^{29,39,41}$. Novamente as metanálises no assun- to ${ }^{34,35,38,42,43}$ não mostraram diferença significativa entre os dois grupos quanto a reoperações, mesmo com recidivas mais frequentes no grupo de hemorroidopexia.

\section{CONCLUSÕES}

Neste estudo, o método mais utilizado para o tratamento cirúrgico das hemorroidas foi a hemorroidectomia convencional. Observou-se ainda que a hemorroidopexia mecânica apresentou tempo operatório mais curto e recuperação pós-operatória mais breve (alta ambulatorial mais precoce). Além disso, houve maior tendência a complicações tardias e recidivas, com necessidade de reintervenção cirúrgica, no grupo de hemorroidectomia convencional, no entanto sem significância estatística.

Conclui-se que tanto a técnica de hemorroidectomia convencional quanto o PPH são boas alternativas para o tratamento cirúrgico da doença hemorroidária.

\footnotetext{
ABSTRACT: The aim of this study was to review the experience of the Coloproctology Department of HSPM-SP in the treatment of hemorrhoids. We have retrospectively studied 253 patients submitted to the surgical treatment of hemorrhoids between $05 / 10 /$ 2004 and 12/31/2007. Patients were distributed in groups: Group 1) Conventional Hemorrhoidectomy (212); Group 2) Stapled Hemorrhoidopexy (41). Epidemiology, indications, results and complications were studied based on registers. From 253 patients studied, 170 were women, with a mean age of 50 years. Mean operating time was 52 minutes (25 -120) in group 1 and 31 minutes $(20-65)$ in group 2 ( $p=0.0001)$. Mean time for ambulatory discharge was 10 and 6 weeks for groups 1 and 2 respectively (p=0.021). The overall complications totalized 28 cases in group 1 and 3 cases in group 2 (p>0,05). In consequence of these complications, 13 cases in group 1, and 1 case in group 2 were submitted to a new surgical procedure. At this Department the most used technique was conventional hemorrhoidectomy. Stapled hemorrhoidopexy was associated with less operating time and a faster functional recovery (early ambulatory discharge). Hemorrhoidectomy was associated with more complications and recurrence, but without statistical significance.
}

Key words: Hemorrhoids, hemorrhoidectomy, hemorrhoidopexy, PPH, complications.

\section{REFERÊNCIAS}

1. Corman ML. Colon and Rectal Surgery, 5th Edition. Lippincott Williams \& Wilkins, 2005:178.

2. Cruz MG et col. Complicações pós-operatórias cirúrgicas da hemorroidectomia: revisão de 76 casos de complicações. Rev Bras Colo-Proct 2007;27(1):42-57.

3. Gordon PH, Nivatvongs S. Principles and Practice for the Surgery of Colon, Rectum and Anus, $3^{\text {rd }}$ ed, Informa Healthcare, 2007:143.

4. Wolff BG, Fleshman JW, Beck DE, Pemberton JH, Wexner SD. The ASCRS Textbook of Colon and Rectal Surgery. Springer, 2007:156.
5. Bacon HE. Anus, rectum and sigmoid colon, 3rd ed, vol 1. Philadelphia: JB Lippincott, 1949:453.

6. Milligan ET, Morgan CN, Jones LE. Surgical anatomy os the anal canal and the operative treatment of hemorrhoids. Lancet 1937;2:119-124.

7. Ferguson JA \& Heaton JR. Closed Hemorrhoidectomy. Dis Colon Rectum 1959;2:176-179.

8. Whitehead W. The surgical treatment of hemorrhoids. Br Med J 1882;1:148-50.

9. Obano RN. Hemorrhoids. An Alap 1966;1:110. 
10. Parks AG. The surgical treatment of haemorrhoids. Br J Surg 1956;43:337-351.

11. Borba MR, Sobrado Jr CW et Sokol S. Hemorroidectomia pela técnica fechada (Técnica de Sokol). - análise de 322 doentes. Rev Bras Colo-Proct 1997;17(2):98-100.

12. Ruiz Moreno. Hemorroidectomia in: $21^{\circ}$ Congresso Brasileiro de Coloproctologia 1971.33.

13. Bleday R, Pena JP, Rothenberger DA, Goldberg SM, Buls JG. Symptomatic hemorrhoids: current incidence and complications of operative therapy. Dis Colon Rectum 1992;35(5):477-481.

14. Eu KW, Seow-Choen F, Goh HS. Comparison of emergency and elective haemorrhoidectomy. Br J Surg 1994;81(2):308310 .

15. Habr-Gama A et col. Surgical treatment of anal stenosis: assessment of 77 anoplasties. Clinics 2005;60(1):17-20.

16. Johnstone CS, Isbister WH. Inpatient management of piles: a surgical audit. Aust N Z J Surg 1992;62(9):720-724.

17. Lacerda-Filho A, Cunha-Melo JR. Outpatient haemorrhoidectomy under local anaesthesia. Eur J Surg 1997;163(12):935-940.

18. Leff EI. Hemorrhoidectomy-laser vs. nonlaser: outpatient surgical experience. Dis Colon Rectum 1992;35(8):743-746.

19. Reis Neto JÁ, Quilici FA, Reis Jr JÁ. Classificação e tratamento das estenoses anais cicatriciais. Rev Bras Colo-Proct 1987;7(1):7-12.

20. Reis Neto JA, Quilici FA, Cordeiro F, Reis Junior JA. Open versus semi-open hemorrhoidectomy: a random trial. Int Surg 1992;77(2):84-90.

21. Sayfan J. Complications of Milligan-Morgan hemorrhoidectomy. Dig Surg 2001;18:131-133.

22. Tagliolatto Jr L. Estenose anal cicatricial: anoplastia por deslizamento de duplo retalho. Rev Bras Colo-Proct 1989;9(4):146-150.

23. Tajana A. Hemorrhoidectomy according to Milligan-Morgan: ligature and excision technique. Int Surg 1989;74(3):158161.

24. Wang JY, Chang-Chien CR, Chen JS, Lai CR, Tang RP. The role of lasers in hemorrhoidectomy. Dis Colon Rectum 1991;34(1):78-82

25. Banov L Jr, Knoepp LF Jr, Erdman LH, Alia RT. Management of hemorrhoidal disease. J S C Med Assoc 1985;81(7):398-401.

26. Longo A. Treatment of hemorrhoids disease by reduction of mucosa and hemorrhoidal prolapse with circular suturing device: a new procedure. In Proceeding of the 6th World Congress of Endoscopic Surgery - Rome. Monduzzi Editore: Bologna 1998; 777-784.

27. Corman ML et al. Stapled haemorrhidopexy: a consensus position paper by an international working party indications, contra-indications and technique. Colorectal Dis 2003:5:304-10.
28. Almeida MG, et al. Tratamento cirúrgico de hemorróidas internas e prolapso mucoso do reto pela técnica de Longo (grampeamento circular) - experiência inicial. Rev Bras ColoProct 2004;24(3):225-229.

29. Brusciano MD, et al. Reinterventions after complicated or failed stapled hemorrhoidopexy. Dis Colon Rectum 2004;47:1846-1851.

30. Cataldo P, et al. Practice parameters for the management of hemorrhoids. Dis Colon Rectum 2005;48:189-194.

31. Ganio E, Altomare DF, Milito G, Gabrielli F, Canuti S. Longterm outcome of a multicentre randomized clinical Trial of stapled haemorrhoidectopexy versus Milligan-Morgan haemorhoidectomy. Br J Surg 2007;94:1033-1037.

32. Gravié JF, et al. Stapled hemorrhoidopexy versus MilliganMorgan hemorrhoidectomy. A prospective, randomized, multicenter trial with 2 year postoperative follow up. Ann Surg 2005;242(1):29-35.

33. Hetzer FH, et al. Stapled vs excision hemorrhoidectomy. Arch Surg 2002;137:337-340.

34. Jayaraman S, Colquhoun PHD, Malthaner RA. Stapled versus conventional surgery for hemorrhoids. Cochrane Database of Systematic Reviews 2006, Issue 4. Art. No.: CD005393.

35. Jayaraman S, Colquhoun PHD, Malthaner RA. Stapled hemorrhoidopexy is associated with a higher long-term recurrence rate of internal hemorrhoids compared with conventional excisional hemorrhoid surgery. Dis Colon Rectum 2007;50:1297-1305.

36. Mattana MD, et al. Stapled hemorrhoidopexy and MilliganMorgan hemorrhoidectomy in the cure of fourth-degree hemorrhoids: long-term evaluation and clinical results. Dis Colon Rectum 2007;50:1770-1775.

37. Ortiz H, Marzo J, Armendáriz P, De Miguel M. Stapled hemorrhoidpexy vs. Diathermy excision for fourth degree hemorrhoids: a randomized clinical Trial and review of literature. Dis Colon Rectum 2005;48:809-815.

38. Pasha J, et al. Stapled hemorrhoidopexy compared with conventional hemorrhoidectomy: systematic review of randomized, controlled trials. Dis Colon Rectum 2004;47:18371845.

39. Raahave D, Jepsen LV, Pedersen IK. Primary and repeated stapled hemorrhoidopexy for prolapsing hemorrhoids: follow-up to five years. Dis Colon Rectum 2008;51:334341.

40. Racalbuto A, et al. Hemorrhoidal stapler prolapsectomy vs. Milligan Morgan hemorrhoidectomy: a long-term randomized trial. Int J Colorectal Dis 2004;19:239-244.

41. Ravo B, et al. Complications after stapled hemorhoidectomy: can they be prevented? Tech Coloproctol 2002;6:83-88.

42. Shao WJ, et al. Systematic review and meta-analysis of randomized controlled trials comparing stapled haemorrhoidopexy with conventional haemorrhoidectomy. $\mathrm{Br}$ J Surg 2008;95:147-160. 
43. Tjandra JJ, Miranda KY, Chan MBBS. Systematic review on the procedure for prolapse and hemorrhoids (stapled hemorrhoidopexy). Dis Colon Rectum 2007;50:878-892.

44. Musiari JP. Estenosis de ano. Sem Méd 1954;104:803-4.
Endereço para Correspondência: RAPHAEL MARIANELLI

R. Castro Alves, $n^{\circ} 265$, apt. 66, Aclimação São Paulo / SP - CEP: 01532-001

Telefone: (0xx11) 9960-4030

E-mail: raphaelmarianelli@yahoo.com.br 